\section{ACKNOWLEDGMENTS}

The following individuals contributed to the development of the 2008 preliminary concept paper entitled "Pharmacokinetics in Patients with Impaired Renal Function—Study Design, Data Analysis and Impact on Dosing and Labeling" and/or a draft guidance, based on the preliminary concept paper, to be published in 2009: Sophia Abraham, Sandhya Apparaju, Gilbert Burckart, Shiew-Mei Huang, Lawrence J. Lesko, Nitin Mehrotra, Janet Norden, Mimi Phan, Sarah Robertson, John Strong, Robert Temple, Ta-Chen Wu, Shen Xiao, Nancy Xu, Lei Zhang, Yuanchao Zhang, and Ping Zhao. Arthur Atkinson, Candace Lee, J. Steve Leeder, Michael Neely, and Kenneth E. Thummel contributed to the working group discussion while at the FDA on sabbatical.

\section{CONFLICT OF INTEREST}

The authors declared no conflict of interest.

○) 2009 ASCPT

1. Huang, S.-M. \& Temple, R. Is this the drug or dose for you?: impact of ethnic factors in global drug development, regulatory review, and clinical practice. Clin. Pharmacol. Ther. 84, 287-294 (2008).

2. US Food and Drug Administration. Guidance for Industry: Pharmacokinetics in Patients with Impaired Renal Function—Study Design, Data Analysis and Impact on Dosing and Labeling $<$ http://www.fda.gov/downloads/Drugs/ GuidanceComplianceRegulatorylnformation/ Guidances/ucm072127.pdf> (14 May 1998).

3. Zhang, Y.D., Zhang, L., Abraham, S., Apparaju, S., Wu, T.-C., Strong, J. et al. Assessment of the impact of renal impairment on systemic exposure of new molecular entities: evaluation of recent new drug applications. Clin. Pharmacol. Ther. 85, 305-311 (2009).

4. Ibrahim, S., Honig, P., Huang, S.-M., Lesko, L. \&Williams, R. Clinical pharmacology studies in patients with renal impairment (RI): past experience and regulatory perspectives, $J$. Clin. Pharmacol. 40, 31-38 (2000).

5. Nolin, T.D., Naud, J., Leblond, F.A. \& Pichette, V. Emerging evidence of the impact of kidney disease on drug metabolism and transport. Clin. Pharmacol. Ther. 83, 898-903 (2008).

6. US Food and Drug Administration. Preliminary Concept Paper: Pharmacokinetics in Patients with Impaired Renal Function - Study Design, Data Analysis and Impact on Dosing and Labeling <http://www.fda.gov/ohrms/dockets/ ac/08/briefing/2008-4351b1-01-FDA.pdf> (19 March 2008).

7. US Food and Drug Administration. Pharmaceutical Sciences Advisory Committee Meeting <http://www.fda.gov/ohrms/dockets/ ac/08/slides/2008-4351s2-00-index.htm> (19 March 2008).

8. Stevens, L.A., Coresh, J., Greene, T., Levey, A.S. Assessing kidney function - measured and estimated glomerular filtration rate. N. Engl. J. Med. 354, 2473-2483 (2006)

9. Levey, A.S., Stevens, L.A., Schmid, C.H., Zhang Y., Castro, A.F., Feldman, H.I. et al. for the CKDEPI (Chronic Kidney Disease Epidemiology Collaboration). A new equation to estimate glomerular filtration rate. Ann. Intern. Med. 150 604-612 (2009).
10. Spruill, W.J.,Wade, W.E. \& Cobb, H.H. Comparison of estimated glomerular filtration rate with estimated creatinine clearance in the dosing of drugs requiring adjustments in elderly patients with declining renal function, $A m$. J. Geriatr. Pharmacother. 6, 153-160 (2008).

11. Stevens, L.A. \& Levey, A.S. Use of the MDRD study equation to estimate kidney function for drug dosing. Clin. Pharmacol. Ther. 86, 465-467 (2009).

12. Spruill, W.J., Wade, W.E. \& Cobb, H.H. III. Continuing the use of the Cockcroft-Gault equation for drug dosing in patients with impaired renal function. Clin. Pharmacol. Ther. 86, 468-470 (2009).

13. US Food and Drug Administration. Guidance for Industry: Exposure-Response Relationships-Study Design, Data Analysis, and Regulatory Applications
$<$ http://www.fda.gov/downloads/Drugs/ GuidanceComplianceRegulatoryInformation/ Guidances/ucm072109.pdf> (April 2003).

14. US Food and Drug Administration. Guidance for Industry: Clinical Studies Section of Labeling for Human Prescription Drug and Biological Products-Content and Format $<$ http://www.fda.gov/downloads/Drugs/ GuidanceComplianceRegulatorylnformation/ Guidances/ucm075059.pdf> (January 2006).

15. US Food and Drug Administration. Draft Guidance for Industry: Clinical Pharmacology Section of Labeling for Human Prescription Drug and Biological Products-Content and Format <http:// www.fda.gov/downloads/Drugs/ GuidanceComplianceRegulatorylnformation/ Guidances/ucm109739.pdf> (February 2009).

\title{
Challenges in Developing Evidence-Based Drug Dosing Guidelines for Adults and Children Receiving Renal Replacement Therapy
}

\author{
BA Mueller ${ }^{1}$ and WE Smoyer ${ }^{2}$
}

The challenges of appropriate drug dosing in patients with renal failure requiring renal replacement therapy (RRT) have been exacerbated by recent trends in both RRT technology and practices. Nearly all these changes have resulted in augmented drug clearance, making most existing RRT drug dosing recommendations obsolete. Many barriers exist to conducting research to update our knowledge of appropriate drug dosing in the context of contemporary RRT. Recommendations on how this research could be conducted, including the use of in vitro techniques, are offered here.

At some point, virtually all clinicians are asked to prescribe drugs to patients with renal failure. Pharmacotherapy is the cornerstone of much of the treatment of these patients. Indeed, the average adult hemodialysis patient takes 10-12 medications daily. ${ }^{1}$ Drug therapy in patients with renal failure is fundamentally different from that in patients with normal renal function for considerations related to pharmacokinetics and renal replacement therapy (RRT). This is true not only for drugs that are removed primarily by the kidneys but also for those that are not. ${ }^{2}$ As a result, patients with renal disease are at increased risk for medication-related

${ }^{1}$ Department of Clinical, Social, and Administrative Sciences, College of Pharmacy, University of Michigan, Ann Arbor, Michigan, USA: ${ }^{2}$ Center for Clinical and Translational Research, The Research Institute at Nationwide Children's Hospital, Columbus, Ohio, USA. Correspondence:WE Smoyer (William.Smoyer@ Nationwidechildrens.org) 
problems. ${ }^{3}$ Appropriate drug dosing is thus a major challenge in these patients, and clinicians look to the research literature to provide guidance. Unfortunately, there is a paucity of published data that can be applied to these dosing situations, and the current, 11-year-old US Food and Drug Administration (FDA) Guidance for Pharmacokinetics in Patients with Impaired Renal Function ${ }^{4}$ has gradually been made obsolete as RRT technology and clinical practices have evolved.

The types and use of RRT have changed dramatically over the last several years, mainly as a result of technological advances. The RRT of the 1980s and 1990s bears little resemblance to today's RRT (Table 1). In nearly all cases, the changes in RRT have resulted in greater drug clearances. The clinical dilemma caused by these advances is that the pharmacokinetic studies that formed the basis for many of the drug dosing recommendations used today were derived in the 1980s and 1990s using RRT techniques and technologies that are no longer used in current practice.

\section{The example of vancomycin} requiring RRT, usually because of the high rate of gram-positive infections in these patients. Because vancomycin is cleared primarily by the kidneys, dosing of this drug must be adjusted in patients receiving RRT. In this setting, serum concentration monitoring can be used to guide these dosing decisions. Early pharmacokinetic studies conducted in the 1980s suggested that vancomycin was not significantly cleared by hemodialysis. Consequently, 1,000 mg of vancomycin was dosed once weekly in hemodialysis

Table 1 Renal replacement therapy trends resulting in augmented drug clearance

\begin{tabular}{ll}
\hline $1990 s$ & 2009 \\
\hline Low-permeability dialyzers & High-permeability dialyzers \\
\hline Dialysis dose not quantified & $\mathrm{Kt} / \mathrm{V}_{\text {urea }}$ target $\geq 1.2$ \\
\hline Dialyzer membranes less biocompatible & Dialyzer membranes more biocompatible \\
\hline Smaller surface area dialyzers & Larger surface area dialyzers \\
\hline Continuous ambulatory peritoneal dialysis & Continuous cycling peritoneal dialysis \\
\hline Lower effluent volume continuous renal & $\begin{array}{l}\text { Higher effluent volume continuous renal } \\
\text { replacement therapies }\end{array}$ \\
\hline replacement therapies &
\end{tabular}

patients, with the dose often given during the last 60-90 minutes of hemodialysis because only a small amount was removed by the dialysis procedure. In marked contrast, contemporary hemodialyzers are highly permeable and quite capable of clearing significant amounts of vancomycin. Today, a 1,000-mg dose of vancomycin once weekly during hemodialysis will almost certainly result in significant underdosing in an adult with renal failure. Similarly, contemporary continuous cycling peritoneal dialysis, using more rapid exchanges and "cyclers" to make these dialysate exchanges, also probably requires different vancomycin dosing regimens than those used with continuous ambulatory peritoneal dialysis. Even original articles describing vancomycin dosing in continuous renal replacement therapy (CRRT) used less efficient hemodiafilters and lower dialysate flow rates than those used today. Consequently, although few data are available, the vancomycin doses needed for higher-volume CRRT are clearly greater than in the past.

Whereas advances in RRT technology and clinical practice have necessitated changes in how vancomycin is dosed, the most recent FDA-approved vancomycin package insert that we could identify makes no mention of dosing recommendations for peritoneal dialysis or CRRT. ${ }^{5}$ Indeed, drug clearance data for hemodialysis or CRRT are not mentioned, and only a recommendation of $1,000 \mathrm{mg}$ every 7-10 days for anuric patients is made. ${ }^{5}$ Clearly, strict adherence to the current vancomycin package insert while treating patients with contemporary RRT would systematically underdose patients and probably jeopardize, rather than enhance, patient safety.
Vancomycin is commonly used in patients

\section{The current dilemma}

The RRT technologies and nephrology practices on which the FDA guidance was based have evolved significantly over the last several years, as the vancomycin example above illustrates. This evolution has created huge knowledge deficits in how to dose drugs appropriately. When we recently co-authored a renal drug dosing text we were concerned to find that CRRT drug dosing studies have been conducted for $<20 \%$ of currently used drugs. ${ }^{6}$ Furthermore, pharmacokinetic studies in newer hybrid RRTs, including slow lowefficiency dialysis and extended daily dialysis, have been conducted for $<1 \%$ of marketed drugs. Importantly, dosing recommendations for one form of RRT usually cannot be applied to other forms of RRT. The cumulative consequence of all of these RRT advances is that clinicians now have dangerously little information to guide the safe and effective dosing of the vast majority of drugs used in patients requiring $\mathrm{RRT}$.

In this context, the vancomycin example above exemplifies a larger problem. Clinicians are very familiar with and knowledgeable about vancomycin, and it has been used for more than 50 years. Were it not for the fact that we can easily measure vancomycin serum concentrations, we would probably not even appreciate how significantly the changes in RRT have influenced vancomycin clearance. More concerning is the fact that clinicians are faced with dosing hundreds of other drugs that have been studied far less than vancomycin and for which serum concentrations cannot be measured in a clinical laboratory. The effects that the recent changes in RRT have had on the pharmacokinetics of these agents are also obviously unknown. Our greatest concern is that there currently is no clear plan to ensure the development of this knowledge, which is critical for the safe and effective use of drugs (both current and future) during RRT. With this in mind, we make the following suggestions to bridge this knowledge gap for pharmacotherapy in patients receiving RRT.

\section{Recommendations}

The 1998 FDA guidance and subsequent preliminary concept paper $^{7}$ provide 
appropriate direction for identifying which drugs should have pharmacokinetic studies conducted in patients with renal disease. Since then, however, both the treatments of acute kidney injury (AKI) and chronic kidney disease and dialysis technologies have changed considerably. Based on these advances, we suggest the following approaches.

Hemodialysis. For drugs likely to be removed by hemodialysis, all hemodialysis pharmacokinetic studies should be conducted using a standardized "dose" of hemodialysis using a $k T / V_{\text {urea }}$ of $\geq 1.2$. In the current guidance, the suggested hemodialysis pharmacokinetic studies do not specify dialysis dose. However, the most recent National Kidney Foundation-Kidney Disease Outcomes Quality Initiative guidelines ${ }^{8}$ identify the dose of hemodialysis that is associated with optimal patient outcomes as a $\mathrm{kT} / \mathrm{V}_{\text {urea }}$ of $\geq 1.2$.

All hemodialysis pharmacokinetic studies should use hemodialyzers of a standardized surface area and ultrafiltration coefficient. Hemodialysis pharmacokinetic studies should be conducted with highpermeability hemodialysis membranes, as are used in the majority of contemporary practice.

All hemodialysis pharmacokinetic studies should be performed in pediatric patients using pediatric hemodialyzers. Such studies should specify the dialyzer surface area (absolute and relative to patient surface area), as well as the hemodialysis dose $\left(\mathrm{kT} / \mathrm{V}_{\text {urea }}\right)$ used.

Continuous renal replacement therapy. For all drugs likely to be used in the critical care environment and likely to have pharmacokinetics affected by CRRT, CRRT pharmacokinetic studies should be performed.

Although many types of CRRT exist, for standardization purposes, all CRRT pharmacokinetic studies should be performed using continuous venovenous hemodialysis (CVVHD). Drug clearance differences at conventional effluent (dialysate + formed ultrafiltrate) rates are usually not substantially different among any of the CRRT modalities for the same total effluent rate. Studies showing differing clearance rates between CVVHD and other forms of CRRT are generally found not to be clinically significantly different

\section{Table 2 Barriers to conducting renal replacement therapy research}

General lack of drug assay availability for overwhelming majority of drugs

No governmental requirement/guidance for pharmaceutical or medical device companies to conduct CRRT pharmacokinetic studies

Funding CRRT pharmacokinetic studies could delay time to market for investigational drugs and devices

Funding CRRT pharmacokinetic studies would increase cost of drug and device development

A requirement of CRRT pharmacokinetic studies for drugs on patent could divert phase IV research funds from other studies

A requirement of CRRT pharmacokinetic studies for generic drugs may fall on manufacturers with limited research and development funds

No readily available research funding source, because this research is not hypothesis driven

Small numbers of CRRT patients at any one site to study

Necessity of multicenter trials

IRB approval across multiple centers is difficult

Increased expense

Subject variability can be large, especially for critically ill subjects

CRRT, continuous renal replacement therapy; IRB, institutional review board.

when total effluent rates are used to generate CRRT drug clearance estimates. Given that CVVHD is the most widely used type of CRRT, pharmacokinetic data from CVVHD would have the broadest applicability to assist clinicians in determining appropriate drug dosing during any type of CRRT with conventional effluent rates.

The dose of delivered therapy for CRRT pharmacokinetic studies should be $35 \mathrm{ml} / \mathrm{h} /$ $\mathrm{kg}$ (or 2,000 $\left.\mathrm{ml} / \mathrm{h} / 1.73 \mathrm{~m}^{2}\right)$. This suggestion is evidence based, because this dose of CRRT has been demonstrated to provide superior patient outcomes in adults. ${ }^{9}$ Although other important trials have shown that lower effluent rates are associated with similar patient outcomes, ${ }^{10}$ RRT doses in this range are currently used widely throughout the world in both adult and pediatric patients. Although some centers use higher or lower rates than this, this rate represents a compromise that will have applicability to most CRRT practices worldwide.

CRRT pharmacokinetic studies should be performed with the most commonly used hemofilters in contemporary practice. There is a wide variety of CRRT hemofilters, and each has different effects on transmembrane drug clearance (from data in our laboratory and others). Thus, to ensure the broadest applicability in practice, CRRT pharmacokinetic studies should be performed using two different CRRT hemo- filters, preferably the two most common in clinical practice at the time. Such studies should specify the dialyzer surface area (absolute and relative to patient surface area) and the ultrafiltration coefficient.

CRRT pharmacokinetic studies should also be performed in pediatric patients using pediatric hemofilters. Although the above suggestions will greatly improve rational drug dosing during CRRT in adults, children with AKI are typically forced to use adult CRRT equipment and adult-sized hemofilters because pediatricspecific equipment is often unavailable. The use of such oversized filters often results in significant distortions in drug pharmacokinetics, particularly in smaller children and infants, and can thus greatly affect both drug efficacy and safety.

\section{Barriers and potential solutions}

The current FDA guidance recommends that pharmacokinetic studies be conducted solely in hemodialysis for selected drugs likely to be cleared by hemodialysis. In light of the markedly increased usage of CRRT, the addition of a request for CRRT drug clearance data and dosing recommendations to the 2008 FDA preliminary concept paper ${ }^{7}$ would significantly improve the safety and efficacy of drugs used in critically ill adults and children with AKI. Ideally, this information would be generated from clinical trials conducted in critically ill adult and pediatric patients. 
Development of CRRT drug dosing recommendations for critically ill children and adults would be challenging, but tens of thousands of patients are already being treated with these modalities annually, largely without any available recommendations to direct their pharmacotherapy. In the absence of pharmacokinetic studies conducted in critically ill patients with AKI, even dosing recommendations based on CRRT clearance estimates derived from in vitro drug clearance studies would be helpful. Those CRRT clearance estimates, combined with knowledge of the complex pharmacokinetic changes that occur in critically ill patients with AKI, would be far superior to what is currently available to clinicians.

Although many thousands of adults and children would benefit from clinical research in this area, many barriers exist to this type of research (Table 2). Despite these barriers, viable alternatives to prospective RRT clinical pharmacokinetic trials already exist. Several solute clearance studies using in vitro hemodialysis and CRRT circuits have shown potential usefulness in developing clinical dosing guidelines. ${ }^{11-13}$ There may be significant advantages of this approach, including elimination of patient risk, reduced time and cost, the ability to easily replicate many types of RRTs, and reduced study administration requirements (e.g., pertaining to institutional review boards, informed consent, and subject recruitment). Application of in vitro findings to known pharmacokinetic parameters in the study population could relatively easily lead to better and safer initial dosing estimates that should then be confirmed in subsequent clinical trials.

\section{Conclusion}

Significant barriers exist to the development of clinical and in vitro studies to enhance evidence-based drug dosing during RRT. However, significant potential opportunities for enhancing their development also exist, including governmental guidance and support to conduct these studies. Implementation of the approaches proposed above by incorporating them into the planned update of the FDA Guidance for Pharmacokinetics in Patients with Impaired Renal Function $^{4}$ would provide vital information to clinicians facing the conundrum of determining appropriate drug dosing in adults and children receiving RRT. In addition, it would almost certainly result in more efficacious and safer pharmacotherapeutic outcomes, as well as more efficient use of health-care resources.

\section{CONFLICT OF INTEREST}

The authors declared no conflict of interest.

(c) 2009 ASCPT

1. Manley, H.J.et al. Medication prescribing patterns in ambulatory haemodialysis patients: comparisons of USRDS to a large not-for-profit dialysis provider. Nephrol. Dial. Transplant. 19, 1842-1848 (2004).

2. Dreisbach, A.W. \& Lertora, J.J. The effect of chronic renal failure on drug metabolism and transport. Expert Opin. Drug Metab. Toxicol. 4, 1065-1074 (2008).
3. Manley, H.J., Cannella, C.A., Bailie, G.R. \& St Peter, W.L. Medication-related problems in ambulatory hemodialysis patients: a pooled analysis. Am. J. Kidney Dis. 46, 669-680 (2005).

4. US Food and Drug Administration. Guidance for Industry: Pharmacokinetics in Patients with Impaired Renal Function-Study Design, Data Analysis and Impact on Dosing and Labeling <http://www.fda.gov/downloads/Drugs/ GuidanceComplianceRegulatoryInformation/ Guidances/ucm072127.pdf> (14 May 1998).

5. Vancomycin hydrochloride (vancomycin hydrochloride for injection) [package insert]. Lake Forest, IL: Hospira, 2004.

6. Aronoff, G.R. et al. Drug Prescribing in Renal Failure: Dosing Guidelines for Adults and Children (Philadelphia, PA: American College of Physicians, 2007).

7. US Food and Drug Administration. Preliminary Concept Paper: Pharmacokinetics in Patients with Impaired Renal Function - Study Design, Data Analysis and Impact on Dosing and Labeling <http://www.fda.gov/ohrms/dockets/ ac/08/briefing/2008-4351b1-01-FDA.pdf> (19 March 2008).

8. National Kidney Foundation-Kidney Disease Outcomes Quality Initiative. Clinical practice guidelines for hemodialysis adequacy, update 2006. Am. J. Kidney Dis. 48 (suppl. 1), S2-S90 (2006).

9. Ronco, C. et al. Effects of different doses in continuous veno-venous haemofiltration on outcomes of acute renal failure: a prospective randomised trial. Lancet 356, 26-30 (2000).

10. Palevsky, P.M., et al. Intensity of renal support in critically ill patients with acute kidney injury. N. Engl. J. Med. 359, 7-20 (2008).

11. Teraoka, T. et al. Prediction of iopromide reduction rates during haemodialysis using an in vitro dialysis system. Nephrol. Dial. Transplant. 20, 754-759 (2005).

12. Stevenson, J.M. et al. Ertapenem clearance during modeled continuous renal replacement therapy. Int. J. Artif. Organs 31, 1027-1034 (2008).

13. Churchwell, M.D., Pasko, D.A., Btaiche, I.F., Jain, J.C. \& Mueller, B.A. Trace element removal during in vitro and in vivo continuous haemodialysis. Nephrol. Dial. Transplant. 22, 2970-2977 (2007). 